\title{
Quarkyonic Chiral Spirals
}

\author{
Toru Kojo ${ }^{\text {a }}$, Yoshimasa Hidaka ${ }^{\mathrm{b}}$, Larry McLerran ${ }^{\mathrm{a}, \mathrm{c}}$, and \\ Robert D. Pisarski ${ }^{\mathrm{c}}$ \\ ${ }^{a}$ RIKEN/BNL Research Center, Brookhaven National Laboratory, \\ Upton, $N Y-11973$, USA \\ ${ }^{\mathrm{b}}$ Department of Physics, Kyoto University, Sakyo-ku, Kyoto 606-8502, Japan \\ ${ }^{\mathrm{c}}$ Department of Physics, Brookhaven National Laboratory, Upton, NY-11973, USA
}

\begin{abstract}
We consider the formation of chiral density waves in Quarkyonic matter, which is a phase where cold, dense quarks experience confining forces. We model confinement following Gribov and Zwanziger, taking the gluon propagator, in Coulomb gauge and momentum space, as $\sim 1 /\left(\vec{p}^{2}\right)^{2}$. We assume that the number of colors, $N_{\mathrm{c}}$, is large, and that the quark chemical potential, $\mu$, is much larger than renormalization mass scale, $\Lambda_{\mathrm{QCD}}$. To leading order in $1 / N_{\mathrm{c}}$ and $\Lambda_{\mathrm{QCD}} / \mu$, a gauge theory with $N_{\mathrm{f}}$ flavors of massless quarks in $3+1$ dimensions naturally reduces to a gauge theory in $1+1$ dimensions, with an enlarged flavor symmetry of $S U\left(2 N_{\mathrm{f}}\right)$. Through an anomalous chiral rotation, in two dimensions a Fermi sea of massless quarks maps directly onto the corresponding theory in vacuum. A chiral condensate forms locally, and varies with the spatial position, $z$, as $\left\langle\bar{\psi} \exp \left(2 i \mu z \gamma^{0} \gamma^{z}\right) \psi\right\rangle$. Following Schön and Thies, we term this two dimensional pion condensate a (Quarkyonic) chiral spiral. Massive quarks also exhibit chiral spirals, with the magnitude of the oscillations decreasing smoothly with increasing mass. The power law correlations of the WessZumino-Novikov-Witten model in $1+1$ dimensions then generate strong infrared effects in $3+1$ dimensions.
\end{abstract}

\section{Introduction}

The phases of Quantum Chromodynamics (QCD) at nonzero temperature and density are a subject of continuing interest. While numerical simulations on the lattice can be of use at nonzero temperature when the quark density is small, standard Monte Carlo techniques are not of use in cold, dense quark matter. 
One expansion which is of utility is to expand in the limit of a large number of colors [1]2]3]4]5]. For cold, dense quark matter - quarks in the fundamental representation, coupled to an $S U\left(N_{\mathrm{c}}\right)$ gauge theory - this gives a "Quarkyonic" phase [6,7,8,9]. Keeping the quark chemical potential, $\mu$, of order one as the number of color $N_{\mathrm{c}} \rightarrow \infty$, the free energy for this phase is dominated by that of quarks. Nonetheless, excitations near the Fermi surface are confined, perhaps baryonic, whence the name.

In this paper, we consider chiral symmetry breaking in Quarkyonic matter. We consider a phenomenological model for confinement, taking the timelike component of the gluon propagator to be $D^{00} \sim 1 /\left(\vec{p}^{2}\right)^{2}$. This is valid in Coulomb gauge, for a spatial momentum $\vec{p}$, and corresponds to a potential which rises linearly in coordinate space. Such a propagator was originally suggested by Gribov [10] and Zwanziger [11. To use such a propagator in cold, dense, quark matter, it is necessary to assume that gluons are insensitive to screening by quarks. For this to be true, the number of flavors, $N_{\mathrm{f}}$, must be $\ll N_{\mathrm{c}}$, and the chemical potential must satisfy $\mu \ll N_{\mathrm{c}}^{1 / 2} \Lambda_{\mathrm{QCD}}$, where $\Lambda_{\mathrm{QCD}}$ is the renormalization mass scale of QCD [6].

Chiral symmetry breaking in such a model has been studied by Glozman and Wagenbrunn [8] and by Guo and Szczepaniak [9], for values of $\mu \sim \Lambda_{\mathrm{QCD}}$. We work in the extreme Quarkyonic limit, $\mu \gg \Lambda_{\mathrm{QCD}}$, so that the effects of chiral symmetry breaking in vacuum can be ignored. It is possible for chiral symmetry breaking to occur at large $\mu$, since we are, by assumption, in a confined regime. Of course there is no guarantee that our results apply to QCD, where $N_{\mathrm{c}}=N_{\mathrm{f}}=3$; nevertheless, there is certainly some range of $N_{\mathrm{c}}, N_{\mathrm{f}}$, and $\mu$, where it does. If applicable to $\mathrm{QCD}$, our results are of interest to intermediate densities, where both conventional nuclear physics and perturbative treatments fail. Notably, this may include the astrophysics of neutron stars.

In vacuum, chiral symmetry breaking occurs through the pairing of a left handed quark with a right handed anti-quark, $\left\langle\bar{\psi}_{R} \psi_{L}\right\rangle \neq 0$, and vice versa. This condensate is, of course, spatially uniform, so that the spontaneous breaking of chiral symmetry does not disturb the Lorentz invariance of the vacuum.

Now consider the effects of a Fermi sea, where there is a net excess of quarks over anti-quarks. The analogy of the usual condensate is illustrated in Fig. 1. Energetically, it costs essentially zero energy to excite a quark right at the edge of the Fermi sea. On the other hand, it costs at least $\sim 2 \mu$ to pull an anti-quark out from deep in the Dirac sea. (Remember that we assume that $\mu$ is very large.) Thus the usual condensate can not be formed spontaneously, and anti-quarks will not enter into our analysis henceforth.

There are numerous features which are not captured by the illustration in Fig. 1. We really should draw not one, but two Fermi seas: one for left handed 


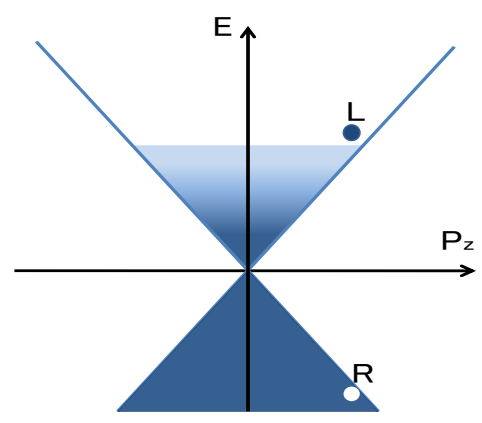

Fig. 1. Quark anti-quark condensate in the presence of a Fermi sea. The energy of the pair is $E_{\text {tot }} \simeq 2 \mu$, while its momentum is $\vec{P}_{\text {tot }} \simeq \overrightarrow{0}$.

quarks, and one for right handed quarks. To avoid unnecessary duplication, instead we assume that the quark, denoted by a filled circle, is always left handed, and that the anti-quark, denoted by an open circle, is right handed. The quark and anti-quark are also assumed to have the same color, so that any condensate is $\sim N_{\mathrm{c}}$, and survives in the limit of large $N_{\mathrm{c}}$. If the quark has momentum $\vec{p}$, then the anti-quark, formed by removing a quark with momentum $\vec{p}$ from the Dirac sea, has momentum $-\vec{p}$. Thus the quark antiquark pair has no net momentum, and this condensate is spatially uniform, as in vacuum.

In the presence of a Fermi sea, though, it is also possible for chiral symmetry to be broken by pairing, say, a (left-handed) quark and a (right-handed) quark hole. If both the quark and the quark hole are near the edge of the Fermi surface, then it costs little energy to excite them, and the energetic penalty paid to excite an antiquark can be avoided. The natural analogy to the condensate in vacuum is illustrated in Fig. 2, pairing a quark with momentum $\vec{p}$, and a hole, formed by removing a quark with momentum $\vec{p}$ from the Fermi surface. The momentum of the hole is then $-\vec{p}$, so the quark-hole pair has no net momentum, and is spatially constant. In condensed matter physics, an excitation as in Fig. 2 is known as an exciton. Naively, we might expect that excitons are suppressed, since the relative momentum between the particle and the hole, $2 \vec{p}$, is large.

However, this is not the only way for quarks and their holes to break the chiral symmetry. Consider pairing a (left handed) quark, with momentum $\vec{p}$, and the hole formed by removing a (right handed) quark with the opposite momentum, $-\vec{p}$, from the Fermi sea. The quark hole then has the same momentum as the quark, $+\vec{p}$, so that the resulting condensate is not uniform, and has a net momentum $+2 \vec{p}$; this is, it varies as $\sim \exp (2 i \mu z)$, where $\hat{z}$ is the direction along which the pair moves, $\vec{p}=p \hat{z}$. Such condensates do not occur in vacuum, where they would imply the spontaneous breaking of rotational symmetry. In condensed matter physics, though, such non-uniform condensates are common, and known as density waves [14]; this is then a chiral density wave. Note that 


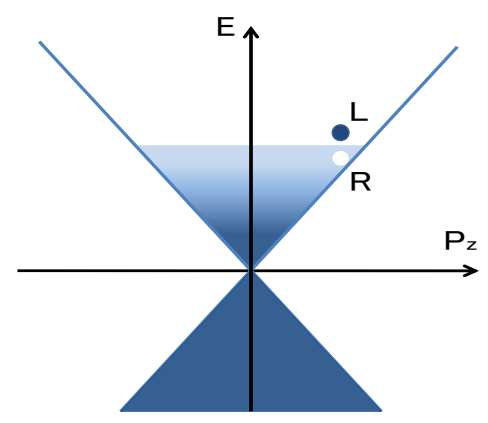

Fig. 2. Exciton pairing between a quark and a quark hole; $E_{\text {tot }} \simeq \vec{P}_{\text {tot }} \simeq \overrightarrow{0}$.

the relative momentum between the quark and its hole is small, so such a condensate may be favored.

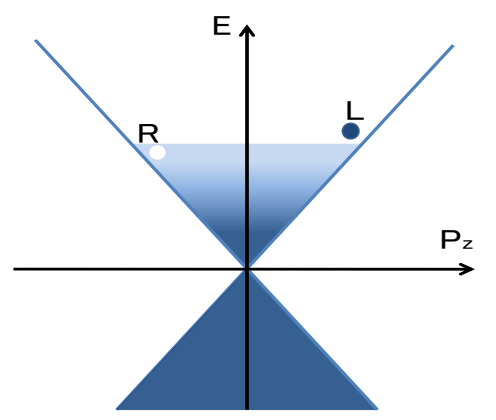

Fig. 3. Pairing between a quark and a quark hole, which generates a chiral density wave; $E_{\text {tot }} \simeq 0, \vec{P}_{\text {tot }} \simeq 2 \mu \hat{z}$.

In this paper we show that in the Gribov-Zwanziger model, that the exciton pairing of Fig. 2 is not generated, but that the chiral density wave of Fig. 3 is. Again, this is familiar from systems in condensed matter: typically excitons are only created dynamically as resonances, such as by the absorption of light, and usually do not condense. Density waves are common, especially for systems in $1+1$ dimensions [14]. We will investigate all Dirac and flavor structures, and show which types are preferred.

For completeness, we illustrate the pairing between two quarks which leads to color superconductivity in Fig. 4. This is pairing between a quark at one edge of the Fermi surface, with momentum $+\vec{p}$, and another quark at the other edge, with momentum $-\vec{p}$. Since pairing is between two quarks, the condensate has no net momentum and is spatially uniform. For this reason, pairing can occur over the entire Fermi surface, in a spatially symmetric state.

So far, we have not emphasized the $N_{\mathrm{c}}$ and $N_{\mathrm{f}}$ dependence of pairing, which is not captured by the illustrations in Figs. 1- 4. The pairing in Figs. 2 and 3 is between a quark and a quark hole of the same color, so the condensate is $\sim N_{\mathrm{c}}$. Further, to the extent that $N_{\mathrm{f}} \ll N_{\mathrm{c}}$, the condensate is rather insensitive to $N_{\mathrm{f}}$. In contrast, the diquark pairing of color superconductivity depends upon 
$N_{\mathrm{c}}$ and $N_{\mathrm{f}}$ in an essential way. Fermi statistics greatly constrains the pairing between two quarks (or two quark holes): it is always anti-symmetric in color, so there are strong relations between the spatial wavefunction, flavor, and chirality. For instance, in case of $N_{\mathrm{f}}=2$ and $N_{\mathrm{c}}=3$, spatially symmetric condensates form by anti-symmetrizing in flavor; this condensate pairs quarks of the same chirality together, and so does not break the chiral symmetry. On the other hand, for $N_{\mathrm{f}}=3$ and $N_{\mathrm{c}}=3$, the preferred condensate does break the chiral symmetry, through color-flavor locking [12].

For more than three colors, the gaps for color superconductivity depend sensitively upon which representation one assumes the quarks to lie in. If the quarks are in the fundamental representation, then since the pairing for color superconductivity is anti-symmetric in the colors of the two quarks, the gap is not a color singlet, and is suppressed at large $N_{\mathrm{c}}$. It is also possible, however, to generalize QCD by letting the quarks lie in the two-index, anti-symmetric representation of color [13. This limit is rather different from that which we consider in this paper. There are $\sim N_{\mathrm{c}}^{2}$ quarks in this limit, so that gluons are affected the quarks, and there is no Quarkyonic phase. This is like taking the number of flavors, $N_{\mathrm{f}}$, to grow with $N_{\mathrm{c}}$. In such a limit color superconductivity is not suppressed at large $N_{\mathrm{c}}$.

It is not clear which of these two limits is most like QCD, with three colors and three light flavors. We suggest that it is useful to consider all possible limits, and to see what qualitative conclusions might be tested in QCD.

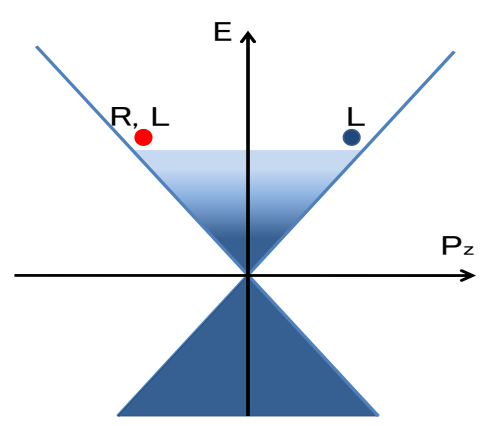

Fig. 4. Pairing between two quarks which generates color superconductivity; $E_{\text {tot }} \simeq \vec{P}_{\text {tot }} \simeq \overrightarrow{0}$.

If a channel for color superconductivity exists, then Cooper pairs will form for arbitrarily weak coupling. Thus color superconductivity is always the dominant pairing mechanism at asymptotically large chemical potential. The essential question is then, how large does the chemical potential have to be for color superconductivity to win out over other pairing mechanisms, such as chiral density waves?

That chiral density waves [14 dominate at large $N_{\mathrm{c}}$ was first demonstrated by Deryagin, Grigoriev, and Rubakov [15, 16, 17, 18, 19, 20, 21, 22, 23, 24]. Using a 
perturbative gluon propagator, $\sim 1 / p^{2}$ in momentum space, they find that chiral density waves form, with a condensate

$$
\Delta_{\text {pert }} \sim \mu \exp \left(-\frac{\pi}{2} \sqrt{\frac{\pi}{\alpha_{s} N_{\mathrm{c}}}}\right)
$$

in magnitude, where $\alpha_{s}=g^{2} / 4 \pi$ is the QCD fine structure constant, measured at a scale $\mu$. Implicitly, the computation assumes that dense quarks form a Fermi liquid, so that pairing is from quarks (and holes) within $\Delta_{\text {pert }}$ of the edge of the Fermi sea.

In contrast, Quarkyonic matter is not a Fermi liquid because of confinement of quarks. Low energy excitations, within $\sim \Lambda_{\mathrm{QCD}}$ of the edge of the Fermi sea, interact not through the perturbative gluon propagator, but through the Gribov-Zwanziger form, $\sim 1 /\left(\vec{p}^{2}\right)^{2}$. A chiral density wave forms, with the same Dirac structure as in perturbation theory. Because the relevant momenta for pairing is controlled by a confining potential, the Quarkyonic condensate for chiral density waves is inevitably $\sim \Lambda_{\mathrm{QCD}}$ in magnitude.

Our analysis applies in a regime of intermediate $\mu$, where the Quarkyonic gap is greater in magnitude than the perturbative gap. we find that for $\Delta_{\text {pert }}<\Lambda_{\mathrm{QCD}}$ whenever $\alpha_{s}>0.12$, using $\Lambda_{\mathrm{QCD}} \sim \mu \exp \left(-6 \pi /\left(11 N_{\mathrm{c}} \alpha_{s}\right)\right)$. For the coupling in QCD, this means that $\mu<80 \mathrm{GeV}$. At larger values of $\mu, \Delta_{\text {pert }}>\Lambda_{\mathrm{QCD}}$. As we discuss at the end of Sec. 2.1, this is a difficult regime to treat, as the effects of both perturbative interactions, and confinement, must be included.

It is known that for a Fermi liquid of dense quarks, chiral density waves lose out to color superconductivity except for very large values of the color, $N_{\mathrm{c}} \geq 1000 N_{\mathrm{f}}$ [16]17]. This is because the chiral density waves generated by perturbative interactions are very sensitive to screening by dynamical quarks.

In sharp contrast, we expect that Quarkyonic chiral density waves are much less sensitive to screening by dynamical quarks. At large $N_{\mathrm{c}}$, dynamical quarks do not affect a Quarkyonic phase until asymptotically large values of the chemical potential, $\mu \sim N_{\mathrm{c}}^{1 / 2} \Lambda_{\mathrm{QCD}}$. This power of $N_{\mathrm{c}}^{1 / 2}$ follows either from considering the Debye mass, as in Ref. [6], or the free energy, as discussed in Appendix A. As long as there is a Quarkyonic phase, and $\Delta_{\text {pert }}<\Lambda_{\mathrm{QCD}}$, we expect that Quarkyonic chiral density waves dominate.

The outline of the paper is as follows. For quark and quark hole excitations near the Fermi surface, where the magnitude of the transverse momentum $\left|\vec{p}_{\perp}\right| \ll \mu$, the theory in $3+1$ dimensions reduces to an effective model in $1+1$ dimensions [14,16]. In Sec. 2 we show that in the Gribov-Zwanziger model, 
this implies that in the gluon propagator, we can integrate over $\vec{p}_{\perp}$ to obtain

$$
\int d^{2} p_{\perp} \frac{1}{\left(p_{0}^{2}-p_{z}^{2}-\vec{p}_{\perp}^{2}\right)^{2}} \sim \frac{1}{p_{0}^{2}-p_{z}^{2}} .
$$

This is the gluon propagator in $1+1$ dimensions, so our effective theory is just QCD in $1+1$ dimensions. We discuss how in the Gribov-Zwanziger model, it is necessary for $\mu \gg \Lambda_{\mathrm{QCD}}$ for this reduction to hold.

In Sec. 3 we consider how quantum numbers in $3+1$ dimensions map onto those in $1+1$ dimensions. Starting with left and right handed massless quarks in $3+1$ dimensions, we find that the reduced model has a doubled flavor symmetry: $N_{\mathrm{f}}$ flavors in $3+1$ dimensions becomes an $S U\left(2 N_{\mathrm{f}}\right)$ symmetry in $1+1$ dimensions. This extended symmetry follows immediately from the analysis of Shuster and Son [16], and is very much like the doubling of flavor symmetry which occurs for heavy quarks.

In Sec. 4 we show how in $1+1$ dimensions, through an anomalous redefinition of the quark fields, a theory at nonzero chemical potential can be mapped onto the corresponding theory in vacuum. The net quark number, present in the theory at $\mu \neq 0$, is generated by the axial anomaly, as shown in Appendix $\mathrm{B}$. The mapping can then be reversed: knowing results for a gauge theory in $1+1$ dimensions in vacuum, one can read off what happens in a Fermi sea, $\mu \neq 0$. We show that a constant condensate in vacuum, $\langle\bar{\psi} \psi\rangle \neq 0$, produces a condensate $\left\langle\bar{\psi} \exp \left(2 i \mu z \Gamma^{5}\right) \psi\right\rangle \neq 0$, where $z$ is the spatial coordinate, and $\Gamma^{5}$ the Dirac matrix in $1+1$ dimensions. In the reduced model, the two dimensional $\Gamma^{5}=\gamma_{0} \gamma_{z}$, where $\gamma_{0}$ and $\gamma_{z}$ are Dirac matrices in $3+1$ dimensions.

This type of spatially dependent condensate at $\mu \neq 0$ is familiar from soluble models in $1+1$ dimensions [18, where Schön and Thies termed it a "chiral spiral" [19]. A chiral spiral was also found by Bringoltz, in his numerical analysis of heavy quarks at nonzero density in QCD in $1+1$ dimensions [20]. We thus term our solution a Quarkyonic Chiral Spiral (QCS).

While we concentrate on massless quarks, in Sec. 5 we discuss how massive quarks also exhibit chiral spirals. This is because even for massive quarks, excitations about the Fermi surface are gapless at tree level.

Effective theories about a QCS are considered in Sec. 6. Using non-Abelian bosonization, the reduced model in $1+1$ dimensions reduces to a Wess-ZuminoNovikov-Witten (WZNW) model [25,26,27]. This model has long range correlations, which should also produce long range correlations in $3+1$ dimensions.

We conclude in Sec. 7 about whether QCS's may be be relevant for cold, dense QCD. We note that QCS's are closely related to pion condensation [22]. They are not identical, because a pion condensate is a chiral spiral in the four 
dimensional $\gamma_{5}$, while for a QCS, it is in the two dimensional $\Gamma^{5}$. We also note that if pionic chiral spirals occur, then probably so do kaonic chiral spirals. As a spatially varying condensate, a kaonic chiral spiral differs for the spatially constant condensate of kaon condensation [23]. Chiral density waves also arise in the Sakai-Sugimoto model [21].

The physics of QCS's should be especially rich, however, since it includes the spontaneous breaking of translational and rotational symmetries, and a plethora of light modes. Such phenomenon should have direct implications for observations of neutron/Quarkyonic stars.

\section{Dimensional Reduction: Self-Consistent Equations}

\subsection{Reduction of the Schwinger-Dyson Equation}

We start by considering the Schwinger-Dyson equation for the quark selfenergy. In a Quarkyonic phase at large $N_{\mathrm{c}}$, the gluons are unaffected by the quarks, so that corrections to the gluon self-energy, and vertices, can be neglected. For the quark self-energy we take the sum of rainbow diagrams,

$$
\mathbb{\&}(p)=-\int \frac{d^{4} k}{(2 \pi)^{4}} D_{\mu \nu}^{A B}(p-k)\left(\gamma_{\mu} t_{A}\right) S(k)\left(\gamma_{\nu} t_{B}\right),
$$

where $\Sigma(p)$ is the quark self-energy. At a nonzero chemical potential $\mu$, the dressed quark propagator, $S(k)$, is

$$
S(k)=\frac{1}{\left[k_{4}+i \mu+\Sigma_{4}(k)\right] \gamma_{4}+\left[k_{j}+\Sigma_{j}(k)\right] \gamma_{j}} .
$$

where $\Sigma$ is determined self-consistently through the integral equation, Eq. (3). We work in Euclidean spacetime, $k=\left(k_{4}, \vec{k}\right)$. In the limit that $\mu \gg \Lambda_{\mathrm{QCD}}$, we neglect chiral symmetry effects as in vacuum, as illustrated in Fig. 1; such effects have been considered at $\mu \neq 0$ by Refs. [8,9]. Consequently, we neglect terms $\sim 1$ in the quark self-energy and propagator.

For the gluon propagator, $D_{\mu \nu}(k)$, we take the Gribov-Zwanziger form,

$$
D_{44}^{A B}(k)=-\frac{8 \pi}{C_{F}} \times \frac{\sigma}{\left(\vec{k}^{2}\right)^{2}} \delta^{A B} \quad ; \quad D^{4 i}=D^{i j}=0 .
$$

At the outset, we stress that we are dealing with a model of confinement. While the gluon propagator, and vertices, are unaffected by quark loops, there is no

fundamental justification in taking the vertices to be the same as the bare 
ones, nor in taking the gluon propagator of Eq. (5). The gluon propagator involves a parameter, $\sigma$, which is the string tension, and has dimensions of mass squared, $\sigma \sim \Lambda_{\mathrm{QCD}}^{2}$. Numerical factors $8 \pi$ and $C_{F}=\left(N_{\mathrm{c}}^{2}-1\right) / 2 N_{\mathrm{c}}$ are multiplied to reproduce a correct linear potential for the color singlet channel.

We stress that the propagator in Eq. (5) is valid only for small momenta, for $k \lesssim \Lambda_{\mathrm{QCD}}$. For larger momenta, one should use the usual gluon propagator of perturbation theory, $\sim 1 / k^{2}$. For excitations near the edge of the Fermi sea, though, we can neglect the perturbative part of the propagator. This differs, for example, from the computation of the free energy [6]. That is dominated by momenta transfers within the entire Fermi sea, $\sim \mu$, for which the perturbative gluon (and quark) propagators should be used. There are contributions to the free energy from momenta $\sim \Lambda_{\mathrm{QCD}}$, but this are small, powers of $\sim\left(\Lambda_{\mathrm{QCD}} / \mu\right)^{2}$ times the perturbative terms [6,7].

After summing over the color indices, Eq. (3) becomes

$$
\mathbb{\&}(p)=\int \frac{d^{4} k}{(2 \pi)^{4}} \frac{8 \pi \sigma}{\left((\vec{p}-\vec{k})^{2}\right)^{2}} \gamma_{4} S(k) \gamma_{4} ;
$$

normalizing the generators as $\operatorname{tr}\left(t^{A} t^{B}\right)=\delta^{A B} / 2$. Since the right hand side of this equation is independent of $p_{4}$, so is the quark self-energy, $\mathbb{\&}(p)$.

The Schwinger-Dyson equation simplifies considerably if we consider only excitations near the edge of a Fermi sea. For a free massless quark with momentum $p^{\mu}=\left(p_{4}, p_{z}, p_{y}, p_{x}\right)=\left(p_{4}, p_{z}, \vec{p}_{\perp}\right)$, in a Fermi sea its mass shell is given by

$$
i p_{4}=\sqrt{p_{z}^{2}+\vec{p}_{\perp}^{2}}-\mu .
$$

Assuming that quark is along the $\hat{z}$ direction, so that $\left|p_{z}\right|=\mu+\delta p_{z}$,

$$
i p_{4} \approx \delta p_{z}+\frac{\vec{p}_{\perp}^{2}}{2 \mu}+\ldots
$$

Thus, as is well known [14], in a Fermi sea the dispersion relation linearizes in $\delta p_{z}$, allowing us to neglect the effects of the transverse momenta for the quarks.

Since this is the mass shell for a free quark, it neglects the effects of the quark self-energy, $\Sigma$. We expect, though, that for quarks and quark holes near the Fermi surface, including the quark self-energy does not affect the suppression of fluctuations in $\vec{p}_{\perp}$. For the Gribov-Zwanziger potential, the natural scale for the transverse momenta is $\left|\vec{p}_{\perp}\right| \sim \Lambda_{\mathrm{QCD}}$. Thus we can neglect the quark transverse momenta in the extreme Quarkyonic limit, where $\mu \gg \Lambda_{\mathrm{QCD}}$. What happens when $\mu \sim \Lambda_{\mathrm{QCD}}$ is a difficult problem which we do not address here.

In the Schwinger-Dyson equation, the dominant contribution from such an 
infrared singular gluon propagator is when the gluon momentum is small. This constrains the internal and external momenta of the quarks to be near one another, $k \approx p$. Neglecting the transverse momenta of the quark, $\vec{k}_{\perp}$, we need only consider the two components of the momenta along the light cone, $k_{z}$ and $k_{4}$. The Schwinger-Dyson equation thus reduces to

$$
\mathbb{\&}(p) \simeq \int \frac{d^{4} k}{(2 \pi)^{4}} \gamma_{4} S\left(k_{4}, k_{z}, \overrightarrow{0}_{\perp}\right) \gamma_{4} \frac{8 \pi \sigma}{\left(\left(\vec{k}_{\perp}-\vec{p}_{\perp}\right)^{2}+\left(k_{z}-p_{z}\right)^{2}\right)^{2}}
$$

Since the transverse momentum $\vec{k}_{\perp}$ enters only through the gluon propagator, we can now integrate it out,

$$
\int \frac{d^{2} k_{\perp}}{(2 \pi)^{2}} \frac{8 \pi \sigma}{\left(\left(\vec{k}_{\perp}-\vec{p}_{\perp}\right)^{2}+\left(k_{z}-p_{z}\right)^{2}\right)^{2}}=\frac{N_{\mathrm{c}} g_{2 \mathrm{D}}^{2}}{2} \frac{1}{\left(k_{z}-p_{z}\right)^{2}} \quad ; \quad N_{\mathrm{c}} g_{2 \mathrm{D}}^{2}=4 \sigma
$$

where we define a two dimensional gauge coupling constant $g_{2 \mathrm{D}}$, and neglect $\vec{p}_{\perp}$.

The reduced Schwinger-Dyson equation then becomes

$$
\mathbb{\&}\left(p_{4}, p_{z}, \overrightarrow{0}_{\perp}\right) \simeq \frac{N_{\mathrm{c}} g_{2 \mathrm{D}}^{2}}{2} \int \frac{d k_{4} d k_{z}}{(2 \pi)^{2}} \gamma_{4} S\left(k_{4}, k_{z}, \overrightarrow{0}_{\perp}\right) \gamma_{4} \frac{1}{\left(k_{z}-p_{z}\right)^{2}}
$$

This integral equation corresponds to QCD in $1+1$ dimensions. In axial gauge, $A_{z}=0$, the two dimensional action for gluons reduces to a free term,

$$
\frac{1}{2} \operatorname{tr} G_{\mu \nu}^{2}=\operatorname{tr}\left(\partial_{z} A_{4}\right)^{2}
$$

In the limit of large $N_{\mathrm{c}}$, the model in $1+1$ dimensions obviously gives the integral equation of Eq. (11).

Of course it is necessary to take some care in the reduction of the Dirac matrices from $3+1$ to $1+1$ dimensions. We address this in Sec. 3 .

A more realistic model for the gluon propagator than Eq. (5) is to take a sum of the Gribov-Zwanziger term, $\sim 1 /\left(\vec{k}^{2}\right)^{2}$, plus a perturbative piece, $\sim 1 / k^{2}$. The confining term is valid for momenta $\lesssim \Lambda_{\mathrm{QCD}}$; the perturbative term, for momenta $>\Lambda_{\mathrm{QCD}}$. The analysis goes through as above. The dependence of the quark propagators on the transverse momenta can be neglected, so one is left with an integral of the gluon propagator with respect to $\vec{k}_{\perp}$. Integration over the Gribov-Zwanziger propagator gives $\sim \sigma /\left(k_{z}-p_{z}\right)^{2}$, while the integral over the perturbative piece generates a logarithm [16,17], $\sim C_{F} g^{2} \log \left(\mu^{2} /\left(k_{z}-p_{z}\right)^{2}\right)$.

With only a perturbative propagator, $\sigma=0$, the analysis is as follows [15,16]17: Assuming that the $\Sigma_{m}$ is a constant gap, $\Delta_{\text {pert }}$, the dimensional reduction 
can apply to the region of quark momenta, $\Delta_{\text {pert }}^{2} / \mu \ll\left|\delta k_{z}\right| \ll \Delta_{\text {pert }}\left(\delta k_{z}\right.$ is measured from the Fermi momentum). Integrating over $k_{4}$ of a quark propagator yields a factor of the inverse energy, $1 /\left|\delta k_{z}^{2}+\Delta_{\text {pert }}^{2}\right|^{1 / 2}$. The form of self-consistent equation has a similar structure as the BCS gap equation for the constant gap. A dominant contribution to the integral comes from the inverse energy part, which is sensitive to the gap (an absence of gap yields infrared divergence in the integrand. This is nothing but the instability of the Fermi surface.) Integration over $k_{z}$ at soft momentum region gives a logarithm term, $\sim \log \left(\mu / \Delta_{\text {pert }}\right)$. Combining it with the logarithm of the gluon propagator, one find the squared logarithmic form of the gap equation, $\Delta_{\text {pert }} \sim g^{2} N_{\mathrm{c}} \log ^{2}\left(\mu / \Delta_{\text {pert }}\right) \Delta_{\text {pert }}$, with the self-consistent solution of Eq. (1).

When we simply add the confining interaction to the self-consistent equation, its nature strongly changes. The self-energy can be no longer identified as the gap from the symmetry breaking, because the single-excitation-energy gap due to the confinement potential is large or divergent. The integral over $k_{z}$ in the gap equation is $\int d k_{z} 1 /\left|\delta k_{z}^{2}+\Sigma_{m}^{2}\right|^{1 / 2} \times \sigma /\left(\delta k_{z}-\delta p_{z}\right)^{2} \sim \sigma / \Lambda_{\mathrm{IR}} \times$ $f\left(\delta p_{z}\right)$, where $f\left(\delta p_{z}\right)$ is a some regular function and $\Lambda_{\mathrm{IR}}$ is an infrared cutoff. The infrared cutoff $\Lambda_{\mathrm{IR}}$ for the gap equation for the single particle spectrum should be identified with the Debye mass, so that the gap itself is of order $\sqrt{N_{\mathrm{c}}} \Lambda_{\mathrm{QCD}}^{2} /\left(\sqrt{g^{2} N_{\mathrm{c}}} \mu\right)$. To see this imagine we try to ionize a hadronic state into its constituent single particle quark excitations. This can only happen when the separation between the constituents is of order the Debye screening length, and costs an energy $\sigma R_{\text {Debye }}=\sqrt{N_{\mathrm{c}}} \Lambda_{\mathrm{QCD}}^{2} /\left(\sqrt{g^{2} N_{\mathrm{c}}} \mu\right)$. This provides a gauge invariant definition for the single particle excitation gap. It also shows that the gap is large compared to the confinement scale in the Quarkyonic Phase. There is no weakly coupled solution for the gap equation when in this phase since, if one goes back to the derivation of the perturbative contribution, the derivation of the logarithmic terms is no longer valid. The dominant contribution for the perturbative piece in arises for momenta greater than the gap itself.

It is important to understand that the analysis here concerns only the single particle excitation spectrum. The issue of chiral condensation is not directly related. We have provided a self-consistent derivation of the chiral condensate in other parts of this paper, and the condensate arises from non-perturbative effects. In a self-consistent analysis of course one cannot rule out the possibility that there is another solution arising from a different kinematic region.

As discussed in the Introduction, we consider only a region of intermediate $\mu$, where the $\Delta_{\text {pert }}<\Lambda_{\mathrm{QCD}}$. In this region, it is safe to include only the confining propagator, and neglect the perturbative term. When $\Delta_{\text {pert }}>\Lambda_{\mathrm{QCD}}$, we enter a more complicated regime, as the large difference in momentum scales required by the perturbative dimensional reduction, between $\Delta_{\text {pert }}^{2} / \mu$ and $\Delta_{\text {pert }}$, is manifestly affected by confinement. Confinement can probably be neglected when $\Lambda_{\mathrm{QCD}}<\Delta_{\text {pert }}^{2} / \mu$, but this only occurs when $\alpha_{s} \sim 0.03$, 
which is an astronomically large value of $\mu, \sim 10^{11} \mathrm{GeV}$.

\subsection{Reduction of the Bethe-Salpeter Equation}

In this subsection we show how the reduction of the Schwinger-Dyson equation for the quark propagator applies as well to the Bethe-Salpeter equation for mesonic wave functions. Henceforth we work in Minkowski spacetime, $k=$ $\left(k_{0}, \vec{k}\right)$. We consider the homogeneous equation, which in large $N_{\mathrm{c}}$ is of the ladder type,

$\widetilde{\Psi}(P ; q)_{\alpha \beta}^{a b}=-\int \frac{d^{4} k}{(2 \pi)^{4}}\left[S(k-P)\left(\gamma^{\mu} t_{A}\right) \widetilde{\Psi}(P ; k)\left(\gamma^{\nu} t_{B}\right) S(k+P)\right]_{\alpha \beta}^{a b} D_{\mu \nu}^{A B}(k-q)$

$a$ and $b$ denote color indices, $\alpha$ and $\beta$ spinor indices. $\widetilde{\Psi}(P ; q)$ is a bound state wave function, where $2 P$ is the total momentum, $q$ the relative momentum. The dressed quark propagator, $S(q)$, is the self-consistent solution to the Schwinger-Dyson equation, Eq. (11). The Bethe-Salpeter equation includes both singlet and adjoint channels, $\Psi^{a b}=\delta^{a b} \Psi+t_{A}^{a b} \Psi_{A}$; for the singlet channel, this reduces to $\left(D_{00}=-D_{44}\right)$

$$
\Psi(P ; q)_{\alpha \beta}=-\int \frac{d^{4} k}{(2 \pi)^{4}}\left[S(k-P) \gamma^{0} \Psi(P ; k) \gamma^{0} S(k+P)\right]_{\alpha \beta} \frac{8 \pi \sigma}{\left((\vec{k}-\vec{q})^{2}\right)^{2}} .
$$

As in the previous subsection, we concentrate on very soft gluons, $\vec{k} \approx \vec{q}$. We assume that the both quarks, with momenta $\vec{k}-\vec{P}$ and $\vec{k}+\vec{P}$ are close to the Fermi momentum, $\left|\vec{p}_{F}\right|=\mu$. We assume that the quark and quark hole pair have a total momentum along the $z$-direction, $2 \vec{P}=2 P \hat{z}$, and that the transverse momentum of the wavefunction can be neglected. We consider pairing energies that are both of the exciton type, $P_{z} \simeq 0$ and $\left|k_{z}\right| \simeq p_{F}$, and for a chiral density wave, $\left|P_{z}\right| \simeq p_{F}$ and $k_{z} \simeq 0$.

Neglecting the transverse momenta of the quark and quark hole, as we did for the quark mass shell in Eq. (8), we then introduce a wavefuntion, $\varphi$, which is obtained by averaging $\Psi(P ; q)$ over the relative, transverse momenta, $\vec{q}_{\perp}$ :

$$
\varphi\left(P ; q_{0}, q_{z}\right)=\int \frac{d^{2} \vec{q}_{\perp}}{(2 \pi)^{2}} \Psi\left(P ; q_{0}, q_{z}, \vec{q}_{\perp}\right) .
$$

The Bethe-Salpeter equation satisfied by this averaged wavefunction is

$$
\begin{aligned}
\varphi\left(P ; q_{0}, q_{z}\right)_{\alpha \beta}=-\int & \frac{d^{2} \vec{q}_{\perp}}{(2 \pi)^{2}} S(q-P)_{\alpha \gamma} S(q+P)_{\delta \beta} \\
& \times \int \frac{d^{4} k}{(2 \pi)^{4}}\left[\gamma^{0} \Psi(P ; k) \gamma^{0}\right]_{\gamma \delta} \frac{8 \pi \sigma}{\left((\vec{k}-\vec{q})^{2}\right)^{2}} .
\end{aligned}
$$


We take the momenta of the quarks to be near the Fermi surface, $\vec{q} \pm \vec{P}=$ $\left(p_{F} \pm \delta p_{z}, \pm \delta \vec{p}_{\perp}\right)$. Thus we introduce two dimensional quark propagators,

$$
\bar{S}(P \pm q) \equiv S\left(P_{0} \pm q_{0}, P_{z} \pm q_{z}, \overrightarrow{0}_{\perp}\right)
$$

The Bethe-Salpeter equation becomes

$$
\begin{aligned}
\varphi\left(P ; q_{0}, q_{z}\right)_{\alpha \beta} \simeq & -\bar{S}(q-P)_{\alpha \gamma} \bar{S}(q+P)_{\delta \beta} \\
& \times \int \frac{d^{4} k}{(2 \pi)^{4}}\left[\gamma^{0} \Psi(P ; k) \gamma^{0}\right]_{\gamma \delta} \int \frac{d^{2} \vec{q}_{\perp}}{(2 \pi)^{2}} \frac{8 \pi \sigma}{\left((\vec{k}-\vec{q})^{2}\right)^{2}}
\end{aligned}
$$

The wave function on the right hand side depends only upon $P$ and $k$, but not upon $q$, and so we can integrate $\Psi(P ; k)$ with respect to $\vec{k}_{\perp}$. That implies that as for the quark self-energy, we can integrate over $\vec{q}_{\perp}$, leaving

$$
\begin{aligned}
\varphi\left(P ; q_{0}, q_{z}\right)_{\alpha \beta} \simeq & -\frac{N_{\mathrm{c}} g_{2 \mathrm{D}}^{2}}{2} \bar{S}(q-P)_{\alpha \gamma} \bar{S}(q+P)_{\delta \beta} \\
& \times \int \frac{d k_{0} d k_{z}}{(2 \pi)^{2}}\left[\gamma^{0} \varphi(P ; k) \gamma^{0}\right]_{\gamma \delta} \frac{1}{\left(k_{z}-q_{z}\right)^{2}}
\end{aligned}
$$

This is the same form as the Bethe-Salpeter equation for QCD in two dimensions [4], in $A_{z}=0$ gauge.

\section{The Effective Lagrangian in $1+1$ Dimensions}

In the previous Section we demonstrated how dimensional reduction, from $3+1$ to $1+1$ dimensions, occurs for both the quark self-energy and the BetheSalpeter wave function. In this section we show how this arises at the level of effective Lagrangians. Our discussion elaborates upon that by Shuster and Son [16].

Start with the free quark Lagrangian in $3+1$ dimensions. Since we concentrate on quarks near the Fermi surface, we assume that the spatial momentum is along the $\hat{z}$ direction,

$$
\mathcal{L}_{\text {kin }}^{\text {lightcone }}=i\left(\bar{\psi} \gamma^{0} \partial_{0} \psi+\bar{\psi} \gamma^{z} \partial_{z} \psi\right)
$$

For the Dirac matrices, we take

$$
\gamma^{0}=\left[\begin{array}{ll}
0 & \mathbf{1} \\
\mathbf{1} & 0
\end{array}\right], \quad \gamma^{j}=\left[\begin{array}{cc}
0 & -\sigma^{j} \\
\sigma^{j} & 0
\end{array}\right], \quad \gamma^{5}=\left[\begin{array}{cc}
\mathbf{1} & 0 \\
0 & -\mathbf{1}
\end{array}\right]
$$

$\sigma^{j}$ are the Pauli matrices, and $\mathbf{1}$ the unit matrix in two dimensions. We also 
define spin matrices as

$$
\Sigma^{i}=\gamma^{5} \gamma^{0} \gamma^{i}=\frac{i \epsilon^{i j k}}{4}\left[\gamma^{j}, \gamma^{k}\right]=\left[\begin{array}{cc}
\sigma^{i} & 0 \\
0 & \sigma^{i}
\end{array}\right]
$$

$\epsilon^{i j k}$ is the totally antisymmetric tensor, $\epsilon^{123}=1$. If transverse momenta can be neglected, the reduced Lagrangian in $1+1$ dimensions has an extended symmetry, which is related to the spin that quarks carry in $3+1$ dimensions.

We introduce projectors for the quark fields,

$$
\psi_{R, L}=\frac{1 \pm \gamma_{5}}{2} \psi ; \psi_{R \pm}=\frac{1 \pm \gamma^{0} \gamma^{z}}{2} \psi_{R} ; \quad \psi_{L \pm}=\frac{1 \pm \gamma^{0} \gamma^{z}}{2} \psi_{L}
$$

$\psi_{R}$ and $\psi_{L}$ are right and left handed fields, eigenstates of chirality. We also introduce projectors for spin [28] along the $\hat{z}$ direction, $\left(1 \pm \Sigma^{z}\right) / 2$; for eigenstates of chirality, this equals $\left(1 \pm \gamma^{0} \gamma^{z}\right) / 2$.

The usual chiral basis is to take

$$
\psi^{T}=\left[\psi_{R+}, \psi_{R-}, \psi_{L-}, \psi_{L+}\right]
$$

but for this problem, this is rather inconvenient. To see this, we write the free Lagrangian in terms of $\psi_{R \pm}$ and $\psi_{L \pm}$,

$$
\begin{aligned}
\mathcal{L}_{\text {kin }}^{\text {lightcone }}= & i\left[\psi_{R+}^{\dagger}\left(\partial_{0}+\partial_{z}\right) \psi_{R+}+\psi_{R-}^{\dagger}\left(\partial_{0}-\partial_{z}\right) \psi_{R-}\right. \\
& \left.+\psi_{L+}^{\dagger}\left(\partial_{0}+\partial_{z}\right) \psi_{L+}+\psi_{L-}^{\dagger}\left(\partial_{0}-\partial_{z}\right) \psi_{L-}\right]
\end{aligned}
$$

The fields $\psi_{R+}$ and $\psi_{L-}$ have spin up along the $+\hat{z}$ direction, while $\psi_{L+}$ and $\psi_{R-}$ have spin along the $-\hat{z}$ direction. In terms of two dimensions, for positive energy $\psi_{R+}$ and $\psi_{L+}$ are right moving fields, while $\psi_{L-}$ and $\psi_{R-}$ are left moving fields.

Because there is no spin in two dimensions, we only need two component spinors. Thus from one four component spinor in $3+1$ dimensions we obtain two types of two component spinors in $1+1$ dimensions,

$$
\varphi_{\uparrow}=\left[\begin{array}{c}
\psi_{R+} \\
\psi_{L-}
\end{array}\right] \quad ; \quad \varphi_{\downarrow}=\left[\begin{array}{c}
\psi_{L+} \\
\psi_{R-}
\end{array}\right] .
$$

The spinors $\varphi_{\uparrow}$ and $\varphi_{\downarrow}$ are eigenstate of spin along the $\hat{z}$ direction, and act as two "flavors" in $1+1$ dimensions. This is valid to the extent that we can neglect the transverse momenta, which couple to the flavor breaking matrix, $\vec{\gamma}_{\perp}$.

We can then combine $\varphi_{\uparrow}$ and $\varphi_{\downarrow}$ into one four component spinor, $\Phi$, that is 
related to the original quark field, $\psi$, by a unitary transform, $U$ :

$$
\Phi=\left[\begin{array}{c}
\varphi_{\uparrow} \\
\varphi_{\downarrow}
\end{array}\right]=U \psi \quad ; \quad U=\left[\begin{array}{cccc}
1 & 0 & 0 & 0 \\
0 & 0 & 1 & 0 \\
0 & 0 & 0 & 1 \\
0 & 1 & 0 & 0
\end{array}\right] \quad ; \quad U^{\dagger} U=1
$$

For the Dirac matrices in two dimensions, $\Gamma^{\mu}, \mu=0, z$, we make the obvious choice,

$$
\Gamma^{0}=\sigma^{1} \quad ; \quad \Gamma^{z}=-i \sigma^{2} \quad ; \quad \Gamma^{5}=\sigma^{3} .
$$

In two dimensions,

$$
\Gamma^{0} \Gamma^{z}=\Gamma^{5} .
$$

While this identity is trivial mathematically, it plays an important role in the next section. The conjugate is defined as $\bar{\Phi}=\left(\bar{\varphi}_{\uparrow}, \bar{\varphi}_{\downarrow}\right)=\left(\varphi_{\uparrow}^{\dagger}, \varphi_{\downarrow}^{\dagger}\right) \Gamma^{0}$.

It is straightforward to rewrite quark bilinears in $3+1$ dimensions in terms of spinors in $1+1$ dimensions. The operators appearing in the action,

$$
\begin{aligned}
& \bar{\psi} \gamma^{0} \psi=\psi_{R+}^{\dagger} \psi_{R+}+\psi_{R-}^{\dagger} \psi_{R-}+(R \leftrightarrow L)=\Phi^{\dagger} \Phi=\bar{\Phi} \Gamma^{0} \Phi ; \\
& \bar{\psi} \gamma^{z} \psi=\psi_{R+}^{\dagger} \psi_{R+}-\psi_{R-}^{\dagger} \psi_{R-}+(R \leftrightarrow L)=\Phi^{\dagger} \Gamma^{5} \Phi=\bar{\Phi} \Gamma^{z} \Phi,
\end{aligned}
$$

directly map from four to two dimensions. Because of these identities, we see that in the presence of gauge fields, and a nonzero chemical potential, that a gauge theory in $3+1$ dimensions maps into one in $1+1$ dimensions,

$$
\mathcal{L}_{\mathrm{eff}}^{2 \mathrm{~d}}=\bar{\Phi}\left[i \Gamma^{\mu}\left(\partial_{\mu}+i g_{2 \mathrm{~d}} A_{\mu}\right)+\mu \Gamma^{0}\right] \Phi-\frac{1}{2} \operatorname{tr} G_{\mu \nu}^{2}
$$

Fermions in two dimensions only require two components. Thus a single, four component spinor in four dimensions becomes two types of two components spinors in two dimensions: $\psi$, or equivalently $\Phi$, become $\varphi_{i}$, where $i=\uparrow, \downarrow$ is the flavor index in two dimensions, generated dynamically by dimensional reduction.

We note that the mass term reduces similarly,

$$
\bar{\psi} \psi=\psi_{R+}^{\dagger} \psi_{L-}+\psi_{L-}^{\dagger} \psi_{R+}+(R \leftrightarrow L)=\bar{\Phi} \Phi
$$

One can write the complete dictionary to go from quark bilinears in $3+1$ dimensions to those in $1+1$ dimensions. We introduce matrices for the two dimensional flavor, which act in the space of $\varphi_{\uparrow}$ and $\varphi_{\downarrow}$ :

$\tau_{f}=\left(\tau_{1}, \tau_{2}, \tau_{3}\right)=U\left(\gamma^{0} \Sigma^{1}, \gamma^{0} \Sigma^{2}, \Sigma^{3}\right) U^{\dagger}=\left(\left[\begin{array}{cc}0 & \mathbf{1} \\ \mathbf{1} & 0\end{array}\right],\left[\begin{array}{cc}0 & -i \mathbf{1} \\ i \mathbf{1} & 0\end{array}\right],\left[\begin{array}{cc}\mathbf{1} & 0 \\ 0 & -\mathbf{1}\end{array}\right]\right)$ 
For operators which are diagonal in flavor,

$$
\bar{\Phi}\left[\begin{array}{cc}
\Gamma^{a} & 0 \\
0 & \Gamma^{a}
\end{array}\right] \Phi=\bar{\psi} \gamma^{0} U^{\dagger}\left[\begin{array}{cc}
\Gamma^{0} \Gamma^{a} & 0 \\
0 & \Gamma^{0} \Gamma^{a}
\end{array}\right] U \psi ;
$$

where $\Gamma^{a}=\mathbf{1}, \Gamma^{5}, \Gamma^{0}$, or $\Gamma^{z}$.

For operators which are not diagonal in flavor, we note that the flavors matrices $\tau_{f}$ do not mix right and left moving components, so $\Gamma^{a}$ and $\tau_{f}$ commute with each other. Thus operators which are not flavor singlets transform as

$$
\bar{\Phi}\left[\begin{array}{cc}
\Gamma^{a} & 0 \\
0 & \Gamma^{a}
\end{array}\right] \tau_{f} \Phi=\bar{\psi} \gamma^{0} U^{\dagger}\left[\begin{array}{cc}
\Gamma^{0} \Gamma^{a} & 0 \\
0 & \Gamma^{0} \Gamma^{a}
\end{array}\right] \tau_{f} U \psi .
$$

The complete list of mapping for quark bilinears is given in Table 1 .

Table 1

Transformation between quark bilinears in $1+1$ and $3+1$ dimensions.

\begin{tabular}{|c|c|c|c|c|}
\hline & $\mathbf{1}$ & $\Gamma^{5}$ & $\Gamma^{0}$ & $\Gamma^{z}$ \\
\hline $\mathbf{1}$ & $\mathbf{1}$ & $\gamma^{0} \gamma^{z}$ & $\gamma^{0}$ & $\gamma^{z}$ \\
$\tau_{1}$ & $-\gamma^{5} \gamma^{1}$ & $-i \gamma^{2}$ & $\gamma^{5} \gamma^{0} \gamma^{1}$ & $-i \gamma^{0} \gamma^{2}$ \\
$\tau_{2}$ & $-\gamma^{5} \gamma^{2}$ & $i \gamma^{1}$ & $\gamma^{5} \gamma^{0} \gamma^{2}$ & $i \gamma^{0} \gamma^{1}$ \\
$\tau_{3}$ & $\gamma^{5} \gamma^{0} \gamma^{z}$ & $\gamma^{5}$ & $-\gamma^{5} \gamma^{z}$ & $-\gamma^{5} \gamma^{0}$ \\
\hline
\end{tabular}

We can also use these results to construct the relevant effective Lagrangian for a model in which the gluon propagator is a sum of a Gribov-Zwanziger term, Eq. (5), and a perturbative term. Integration over the former gives QCD in two dimensions, while integration over the perturbative gluon propagator give a non-Abelian Thirring model [16]. Thus the general effective model is a gauged, non-Abelian Thirring model. As noted in the Introduction, we consider only a region of intermediate $\mu$, where the effects of the Thirring model can be neglected.

\section{Mapping a Fermi Sea of Massless Quarks onto the Vacuum}

In two spacetime dimensions, for massless quarks one can directly map the theory at $\mu \neq 0$ onto that in vacuum, $\mu=0$. This has been noted before, in various guises, in the literature before [18], especially by Christiansen and Schaposnik [18]. Hopefully our discussion adds clarity.

Consider the following transformation of the quark fields:

$$
\Phi=\exp \left(-i \mu z \Gamma^{5}\right) \Phi^{\prime}
$$


This transformation is defined to be the same for each of the two dimensional "flavors", $\varphi_{\uparrow}$ and $\varphi_{\downarrow}$, and so preserves the flavor symmetry. Under this transformation, the Lagrangian becomes

$$
\mathcal{L}_{\text {eff }}^{2 \mathrm{D}}=\bar{\Phi}\left[i \Gamma^{\mu}\left(\partial_{\mu}+i g_{2 \mathrm{D}} A_{\mu}\right)+\mu \Gamma^{0}\right] \Phi=\bar{\Phi}^{\prime}\left[i \Gamma^{\mu}\left(\partial_{\mu}+i g_{2 \mathrm{D}} A_{\mu}\right)\right] \Phi^{\prime}
$$

That is, by redefining the quark fields, we have completely eliminated the chemical potential; one has transformed the theory from one in the presence of a Fermi sea to that in vacuum. This happens because when $\Gamma^{z} \partial_{z}$ acts upon $\exp \left(i \mu z \Gamma^{5}\right)$, it equals $\mu \Gamma^{z} \Gamma^{5}$, which by Eq. (29), equals $-\mu \Gamma^{0}$, and so cancels the term for the quark chemical potential in the original Lagrangian.

As we discuss in Sec. 5, this is special to massless quarks, and does not hold for massive quarks. It also does not hold in higher dimensions, where the effects of transverse fluctuations obviate any such correspondence.

We then have a quandry: there is a nonzero density of quarks in the original theory, with $\mu \neq 0$. The vacuum has no such density, so where did it go? The answer is that the transformation of Eq. (36) is anomalous, involving the Dirac matrix $\Gamma^{5}$.

One can show that the correct quark density is given, precisely, by the anomaly. There are many ways of doing the calculation; in Appendix B we give the computation based upon operator regularization. The computation also shows that the only quark bilinear to receive an anomalous contribution is that for quark number. For other operators, the transformation from $\Phi$ to $\Phi^{\prime}$ can be computed naively.

The most interesting transformation is for the chiral condensate. Using Eq. (32), we write the a chiral condensate for $\Phi$, in terms of $\Phi^{\prime}$ :

$$
\bar{\Phi}^{\prime} \Phi^{\prime}=\cos (2 \mu z) \bar{\Phi} \Phi-i \sin (2 \mu z) \bar{\Phi} \Gamma^{5} \Phi .
$$

Assume that there is chiral symmetry breaking in vacuum, so that $\left\langle\bar{\Phi}^{\prime} \Phi^{\prime}\right\rangle \neq 0$. Actually, in $1+1$ dimensions fluctuations disorder the system, and only leave quasi long range order [14]. Since this is due to fluctuations, at large $N_{\mathrm{c}}$ such disorder only occurs over distances exponential in $N_{\mathrm{c}}$ [26]27]. Neglecting such details, if chiral symmetry breaking occurs in vacuum, then it also occurs in the presence of a Fermi sea, in the following manner:

$$
\langle\bar{\Phi} \Phi\rangle=\cos (2 \mu z)\left\langle\bar{\Phi}^{\prime} \Phi^{\prime}\right\rangle \quad ; \quad\left\langle\bar{\Phi} \Gamma^{5} \Phi\right\rangle=-i \sin (2 \mu z)\left\langle\bar{\Phi}^{\prime} \Phi^{\prime}\right\rangle .
$$

This is the strict analogy of Migdal's pion condensation [22] in 1+1 dimensions. Chiral symmetry is broken, but by a spiral in the two possible directions, between $\bar{\Phi} \Phi$ and $\bar{\Phi} \Gamma^{5} \Phi$. Schön and Thies [19] termed this as a "chiral spiral", and we adopt their evocative name, and so refer to our result as a Quarkyonic Chiral Spiral. 
We can also understand why exciton pairing is not favored. An exciton condensate corresponds to $\psi_{R+}^{\dagger} \psi_{L+}$, which is a spin 1 operator. In the effective theory, the corresponding operators are $\bar{\Phi} \Gamma^{z} \tau_{1,2} \Phi$ and $\bar{\Phi} \Gamma^{0} \tau_{1,2} \Phi$. These operators are flavor non-singlet. In two dimensions in vacuum, though, it is expected that the spontaneous breaking of chiral symmetry proceeds through condensates which are flavor singlets, and not through condensates which carry flavor.

We conclude this section by noting that the extended flavor symmetry in $1+1$ dimensions is special to the vector-like interactions of QCD, and is not a generic property of theories in $3+1$ dimensions. Consider, for example, a general Nambu-Jona-Lasino (NJL) model, with interactions such as $(\bar{\psi} \psi)^{2}$. This is the square of a mass term, and so is not invariant under the anomalous chiral transformation of Eq. (36). In accord with this, Nickel found that in NJL models at nonzero density, the thermodynamically favored ground state is a crystal, but not a chiral spiral [24].

\section{Mapping Fluctuations for a Fermi Sea of Massive Quarks}

In this section we show how for massive quarks, excitations near the Fermi surface can be mapped onto a (modified) theory of the vacuum. For massless quarks, this could be done for the entire theory; here, it is only for fluctuations near the Fermi surface.

Let the quark mass be $m$, so the Fermi momentum is related to the chemical potential as $\mu^{2}=p_{F}^{2}+m^{2}$. We work in the extreme Quarkyonic limit, where $\mu \gg \Lambda_{\mathrm{QCD}}$. Starting with the theory in $3+1$ dimensions, by neglecting the transverse momenta we obtain an effective theory in $1+1$ dimensions,

$$
\mathcal{L}_{\mathrm{eff}}^{2 \mathrm{~d}}=\bar{\Phi}\left[i \Gamma^{\mu}\left(\partial_{\mu}+i g_{2 \mathrm{~d}} A_{\mu}\right)+\mu \Gamma^{0}-m\right] \Phi
$$

In this case, we could perform the transformation of Eq. (36), and so eliminate the term $\sim \mu \Gamma^{0}$ from the action, as in Eq. (37). The chemical potential is still in the action, though, through the transformation of the mass term into a complicated, position dependent "mass", as in Eq. (38).

While one cannot make an exact correspondence to the vacuum theory, one can still make an interesting, if more limited, correspondence. Consider not all fluctuations in the theory, but just those near the Fermi surface. As is well known [14], even massive particles have a massless dispersion near the Fermi surface, with a modification to the speed of light:

$$
p_{0}=\sqrt{\left(p_{F}+\delta p\right)^{2}+m^{2}}-\mu \approx v_{F} \delta p \quad ; \quad v_{F}=\frac{\left|p_{F}\right|}{\mu}
$$


As for the massless case, we can neglect anti-quarks, deep in the Fermi sea.

First, let us decompose the spectrum of quarks. We have two Fermi surfaces, $\pm\left|p_{F}\right|$, and have particle and hole excitations at each Fermi sea, with energy $E \simeq \pm v_{F}|\delta p|$. We identify the excitation around the Fermi sea with $+\left|p_{F}\right|$ as a right moving fermion, and with $-\left|p_{F}\right|$ as a left moving fermion. The effective Lagrangian becomes

$$
\mathcal{L}_{\mathrm{eff}}^{2 \mathrm{~d} \text {,massive }}=\bar{\Phi}\left[i \Gamma^{0}\left(\partial_{0}+i A_{0}\right)+i v_{F} \Gamma^{z} \partial_{z}\right] \Phi
$$

where we have assumed $A_{z}=0$ gauge. This is the same theory as for the massless case, Eq. (37), except the speed of light is not one, but $v_{F}$.

These elementary manipulations can be used to explain the nature of chiral spirals in exactly soluble models in $1+1$ dimensions [19,20].

For massless quarks, one maps the complete theory, in the presence of a Fermi sea, onto the vacuum. Thus the computation of $\left\langle\bar{\Phi}^{\prime} \Phi^{\prime}\right\rangle$ is complete, and both $\langle\bar{\Phi} \Phi\rangle$ and $\left\langle\bar{\Phi} \Gamma^{5} \Phi\right\rangle$ oscillate about zero. There are several examples, such as the Gross-Neveu model for a large number of flavors, in which this can be computed analytically [19].

Bringoltz considered a nonzero density of massive quarks for QCD in $1+1$ dimensions [20]. By numerical analysis of the theory in the canonical ensemble, he showed that chiral spirals also arise for massive quarks. These are due to quark and quark hole excitations about the edge of the Fermi surface, Eq. (42). As the density increases, $v_{F} \rightarrow 1$, and the massive theory approaches the massless limit.

\section{Effective Theories for Excitations about the Fermi Surface}

The massless excitations near the Fermi surface can be described in terms of a Wess-Zumino-Novikov-Witten (WZNW) theory [25]26 27]. The dictionary between bosonic and fermionic currents for color, flavor, and (baryon) charge elements is

$$
\begin{aligned}
& J_{+}^{A}=\frac{i}{2 \pi} \operatorname{tr}\left[h^{-1}\left(\partial_{+} h\right) t_{A}\right]=: \Psi_{+}^{\dagger} t_{A} \Psi_{+}:, J_{-}^{A}=\frac{i}{2 \pi} \operatorname{tr}\left[h\left(\partial_{-} h^{-1}\right) t_{A}\right]=: \Psi_{-}^{\dagger} t_{A} \Psi_{-}: \\
& J_{+}^{f}=\frac{i}{2 \pi} \operatorname{tr}\left[g^{-1}\left(\partial_{+} g\right) \tau_{f}\right]=: \Psi_{+}^{\dagger} \tau_{f} \Psi_{+}:, J_{-}^{f}=\frac{i}{2 \pi} \operatorname{tr}\left[g\left(\partial_{-} g^{-1}\right) \tau_{f}\right]=: \Psi_{-}^{\dagger} \tau_{f} \Psi_{-}: \\
& J_{+}=\sqrt{\frac{N_{\mathrm{c}} N_{\mathrm{f}}}{2 \pi}} \partial_{+} \phi=: \Psi_{+}^{\dagger} \Psi_{+}:, J_{-}=\sqrt{\frac{N_{\mathrm{c}} N_{\mathrm{f}}}{2 \pi}} \partial_{-} \phi=: \Psi_{-}^{\dagger} \Psi_{-}:
\end{aligned}
$$

$t_{A}$ is the color matrix, and $h$ an element of $S U\left(N_{\mathrm{c}}\right) ; \tau_{f}$ is the flavor matrix, and $g$ an element of $S U\left(2 N_{\mathrm{f}}\right)$. Normal ordering of a composite operator $A$ is 
denoted by :A: .

For free Dirac fermions with $N_{\mathrm{c}}$ colors and $2 N_{\mathrm{f}}$ flavors,

$$
S=\int d^{2} x\left[\Psi_{+} i \partial_{-} \Psi_{+}+\Psi_{-} i \partial_{+} \Psi_{-}\right]
$$

the bosonized version is

$$
S=S_{U(1)}[\phi]+S_{2 N_{\mathrm{f}}}^{\text {color }}[h]+S_{N_{\mathrm{c}}}^{\text {flavor }}[g]
$$

with

$$
\begin{aligned}
S_{U(1)}[\phi] & =N_{\mathrm{c}} N_{\mathrm{f}} \int d^{2} x\left(\partial_{\mu} \phi\right)^{2} \\
S_{k}[l] & =k \operatorname{tr}\left[\frac{1}{16 \pi} \int d^{2} x \partial_{\mu} l \partial^{\mu} l^{-1}+\frac{1}{24 \pi} \int d^{3} x \epsilon^{\mu \nu \lambda}\left(l^{-1} \partial_{\mu} l\right)\left(l^{-1} \partial_{\nu} l\right)\left(l^{-1} \partial_{\lambda} l\right)\right] .
\end{aligned}
$$

This is a sum of a free massless scalar, for the $U(1)$ of baryon number, a $S U\left(N_{\mathrm{c}}\right)$ WZNW model with level $2 N_{\mathrm{f}}$, and a $S U\left(2 N_{\mathrm{f}}\right)$ WZNW model with level $N_{\mathrm{c}}$.

A similar form can be derived for the theory of Dirac fermions coupled to a gauge field [26,27]. The flavor part of the action, $S_{N_{\mathrm{c}}}^{\text {flavor }}[g]$, is completely unchanged, because the currents which define the flavor matrix $g$ are color singlets. The color dependent part of the action, $S_{2 N_{\mathrm{f}}}^{\text {color }}[h]$, becomes that of a gauged WZNW action.

The spectrum of a gauged WZNW model is involved [26,27]. However, what we are most concerned about are excitations near the Fermi surface; namely, are there gapless modes. In this context, what is of greatest concern is that is that the massless correlations of the flavor WZNW action, $S_{N_{\mathrm{c}}}^{\text {flavor }}[g]$, dominate correlations over large distances.

Note that this is true for both massless and massive quarks. In either case, there are numerous gapless modes about the Fermi surface.

\section{Conclusions}

In this paper we approximate the confining potential in a Quarkyonic phase as in Eq. (5). We then find that Quarkyonic Chiral Spirals (QCS's) arise naturally, and can be expressed in terms of an effective model in $1+1$ dimensions. Our analysis is valid in the extreme Quarkyonic limit, where $\mu \gg \Lambda_{\mathrm{QCD}}$, within a narrow skin of the surface of the Fermi sea, $\sim \Lambda_{\mathrm{QCD}}$. 
In this work we only considered the formation of a single chiral density wave, in a fixed direction. It is most likely, though, that the entire Fermi surface is covered with patches of chiral density waves, in different directions [16]17]. The detailed manner in which the Fermi surface is covered with such patches will be discussed separately [29].

Quarkyonic Chiral Spirals are reminiscent of the pion condensates of Migdal [22]. Pion condensates arise in effective models of nucleons interacting with pions: they are chiral spirals which oscillate as $\left\langle\bar{\psi} \exp \left(2 i c f_{\pi} z \gamma_{5}\right) \psi\right\rangle$, where $c$ is a pure number and $f_{\pi}$ is the pion decay constant. In contrast, a QCS arises from the interactions of quarks and gluons; it is a chiral spiral not in chirality, $\gamma_{5}$, but in spin, $\gamma^{0} \gamma^{z}$.

It is of great interest that we find a QCS for massive quarks. Kaplan and Nelson suggested that in dense matter, effective models of nucleons and kaons indicate that there is a condensate for the $K^{-}$field [23]. This is constant in space, and so is unlike the chiral spiral which we would expect in strange Quarkyonic matter.

For distances $x \gg 1 / \mu$, QCS's have numerous modes with long ranged correlations, from the correlations of the WZNW model, Sec. 6. It is possible that the long range correlations of the modes of the WZNW model acquired finite range over distances $\sim 1 / \Lambda_{\mathrm{QCD}}$. Even so, this predicts many more light modes than expected otherwise.

The formation of QCS's has strong implications for the phase diagram of a gauge theory. The usual expectation is that one goes from a phase where chiral symmetry breaking is broken through a constant condensate, $\langle\bar{\psi} \psi\rangle \neq 0$, directly to a phase where it vanishes, $\langle\bar{\psi} \psi\rangle=0$.

If a QCS forms, chiral symmetry is broken, but through an order parameter which differs from that in vacuum. As for a pion condensate, a QCS spontaneously breaks both translational and rotational symmetries. Thus there is a strict order parameter which differentiates between ordinary chiral symmetry breaking, with a constant chiral order parameter, and a QCS. It also implies that there are exactly massless Goldstone bosons in a QCS, from the spontaneously broken symmetries of translation and rotation. Similarly, there is an order parameter which differentiates between a QCS, and a phase which is chirally symmetric (at least for massless quarks).

This implies that at zero temperature, as $\mu$ increases there is first a Fermi sea. There is then a well defined phase transition from a phase with constant $\langle\bar{\psi} \psi\rangle \neq 0$ to a QCS. There is then a second phase transition, from the QCS to a chirally symmetry phase, with $\langle\bar{\psi} \psi\rangle=0$. This differs from models which exclude spatially dependent condensates, Ref. [8,9], but similar to models which allow for then, Ref. [24]. 
Thus at $T=0$ and $\mu \neq 0$, we predict the existence of an intermediate phase, with a QCS. In Appendix A we show that quarks affect gluons at asymptotically large $\mu \sim N_{\mathrm{c}}^{1 / 2}$. At such large $\mu$, deconfinement and chiral symmetry breaking surely occur. Thus suggests that at least for large $N_{\mathrm{c}}$, the region with a QCS is also large. Further, it is very possible that the phase transition from a QCS, to a chirally symmetry phase, occurs before the theory deconfines.

We conclude by suggesting that dynamical quarks do not easily wash out a Quarkyonic phase, nor related effects, such as Quarkyonic chiral spirals. In QCD, it is known that the effects of screening, from dynamical quarks, are not strong. Notably, the linear term in the quark anti-quark potential persists to rather short distances, $\sim 0.2 \mathrm{fm}$. This distance is comparable to the short range repulsion experienced by nucleons. Short distances then translates into high densities. It would be very interesting to estimate this effect within effective models, such as approximate solutions to the Schwinger-Dyson equations.

Our analysis clearly raises more questions than it answers. However, we hope that we have provided a different way for thinking about cold, dense quark matter, and about the surprises which it might provide. In the end, this is of direct relevance to neutron (Quarkyonic?) stars, for which a wealth of experimental data will be forthcoming in the next few years.

\section{Acknowledgments}

The research of R. D. Pisarski and L. McLerran is supported under DOE Contract No. DE-AC02-98CH10886. R. D. Pisarski also thanks the Alexander von Humboldt Foundation for their support. This research of Y. Hidaka is supported by the Grant-in-Aid for the Global COE Program "The Next Generation of Physics, Spun from Universality and Emergence" from the Ministry of Education, Culture, Sports, Science and Technology (MEXT) of Japan. T. Kojo is supported by Special Posdoctoral Research Program of RIKEN. L. McLerran gratefully acknowledges conversation with Thomas Schäfer, who insisted that chiral symmetry breaking must occur in the form of chiral den-

sity waves at large $N_{\mathrm{c}}$. We also thank Gokce Basar, Barak Bringoltz, Michael Buchoff, Aleksey Cherman, Thomas Cohen, Gerald Dunne, Robert Konik, Alex Kovner, Dominik Nickel, and Alexei Tsvelik for useful discussions and comments. 


\section{A Screening by Dynamical Quarks at Large $N_{\mathrm{c}}$}

In this appendix we show that at a nonzero temperature, $T$, screening by dynamical quarks enters when $\mu \sim N_{\mathrm{c}}^{1 / 2} T$.

Consider, for example, the square of Debye screening mass at one loop order [6]. Gluons contribute $\sim g^{2} N_{\mathrm{c}} T^{2}$, quarks give $\sim g^{2} N_{\mathrm{f}} \mu^{2}$. Balancing the two terms, quarks start affecting gluons when when $\mu \sim N_{\mathrm{c}}^{1 / 2} T$.

Next consider the same analysis for the free energy. Contributions from gluons are $\sim N_{\mathrm{c}}^{2} T^{4}$, while those from quarks are $\sim N_{\mathrm{c}} N_{\mathrm{f}}$ times powers of $\mu$ and $T: \mu^{4}$, $\mu^{2} T^{2}$, and $T^{4}$, see Eq. A.3 below. Of course at $\sim g^{4}$ and beyond, the quark and gluon contributions get mixed up together, but this does not affect our power counting in $N_{\mathrm{c}}$. If we then balance the leading term for gluons, $\sim N_{\mathrm{c}}^{2} T^{4}$, against that of quarks, $\sim N_{\mathrm{c}} N_{\mathrm{f}} \mu^{4}$, we estimate that quarks dominate when $\mu \sim N_{\mathrm{c}}^{1 / 4} T$ [6], and not $\sim N_{\mathrm{c}}^{1 / 2} T$, as for the Debye mass.

With the free energy, however, one must take more care. One cannot simply equate the magnitude fo the free energies, but remember that deconfinement is only defined by a change in the relevant order parameter, which is the (renormalized) Polyakov loop [30]. This is generated by a nontrivial distribution in the eigenvalues of the thermal Wilson line. This can be modeled by expanding about a constant expectation for the timelike component of the vector potential,

$$
A_{0}=\frac{Q}{g}
$$

$Q$, is a diagonal matrix in color space, and is traceless, as a sum over elements of $S U\left(N_{\mathrm{c}}\right) . Q$ has dimensions of mass, where the mass scale is set by the temperature, $T$. We do not need to know the explicit distribution of the $Q$ 's in order to estimate how large $\mu$ must be for quarks to affect the $Q$-distribution.

At very large $\mu$ we can compute the quark determinant, in the presence of this background field, to one loop order. A constant field for $A_{0}$ acts like an imaginary chemical potential for color, and so it is natural to introduce a color dependent chemical potential [31],

$$
\widetilde{\mu}=\mu+i Q
$$

At one loop order one obtains the usual result for the pressure, with $\mu$ replaced by $\widetilde{\mu}$ :

$$
p_{\text {quark }}=N_{\mathrm{f}}\left(\frac{1}{12 \pi^{2}} \operatorname{tr} \tilde{\mu}^{4}+\frac{T^{2}}{6} \operatorname{tr} \tilde{\mu}^{2}+\frac{7 \pi^{2}}{180} N_{\mathrm{c}} T^{4}\right) .
$$

For large $\mu$, the dominant contribution is from the expansion of the first term, $\sim N_{\mathrm{f}} \operatorname{tr} \widetilde{\mu}^{4} \sim \mu^{4}$, as we estimated above. While this is as large as the gluon 
contribution when $\mu \sim N_{\mathrm{c}}^{1 / 4} T$, that does not matter, since this term is independent of $Q$. This holds order by order in perturbation theory, simply because $Q$ has dimensions of mass. The next term is from the expansion of $\sim N_{\mathrm{f}} \operatorname{tr} \widetilde{\mu}^{4}$, equal to $\sim \mu^{3} \operatorname{tr} Q$; this vanishes, though, because $Q$ is a traceless matrix.

The leading term which is $Q$-dependent is the next term in the expansion of $\operatorname{tr} \widetilde{\mu}^{4}$, which is $\sim \mu^{2} \operatorname{tr} Q^{2}$. Since the trace is $\sim N_{\mathrm{c}}$, this is as large as the gluon contribution, $\sim N_{\mathrm{c}}^{2}$, when $\mu \sim N_{\mathrm{c}}^{1 / 2} T$. This agrees with our estimate from the Debye mass.

At large $N_{\mathrm{c}}$, since the quarks do not affect gluons until $\mu \sim N_{\mathrm{c}}^{1 / 2} T$, the boundary from the confining, to the deconfining, phase is a straight line in the plane of $T$ and $\mu$ [6]. The pure glue theory has a global symmetry of $Z\left(N_{\mathrm{c}}\right)$, which for large $N_{\mathrm{c}}$ is approximately $U(1)$. At large $\mu$, the leading term from quarks is $\sim \operatorname{tr} Q^{2}$, and breaks the $Z\left(N_{\mathrm{c}}\right)$ symmetry. Like other terms from quarks, this favors a real expectation value for the Polyakov loop. Such a term acts to wash out the line of first order transitions.

There are two possibilities. One is that the quarks produce a critical endpoint for deconfinement. The other is that the first line for deconfinement bends and meets the axis for $T=0$. Since all of our analysis depends upon $T \neq 0$, we favor the former.

\section{B Anomalous Baryon Number}

In this appendix we compute the baryon number generated by the anomalous transformation in Eq. (36). Consider the operator for baryon number, computed with point splitting:

$$
\begin{aligned}
& \lim _{\epsilon \rightarrow 0}\left\langle\bar{\Phi}(x+\epsilon) \Gamma^{0} \Phi(x)\right\rangle_{\mu \neq 0}=\lim _{\epsilon \rightarrow 0} \frac{\int \mathcal{D} \bar{\Phi} \mathcal{D} \Phi \bar{\Phi}(x+\epsilon) \Gamma^{0} \Phi(x) e^{i S[\Phi ; \mu \neq 0]}}{Z[\mu \neq 0]} \\
& =\lim _{\epsilon \rightarrow 0} \frac{\int \mathcal{D} \bar{\Phi}^{\prime} \mathcal{D} \Phi^{\prime} \bar{\Phi}^{\prime}(x+\epsilon) e^{i \mu \epsilon_{z} \Gamma^{5}} \Gamma^{0} \Phi^{\prime}(x) e^{i S\left[\Phi^{\prime} ; \mu=0\right]}}{Z[\mu=0]} \\
& =2 \lim _{\epsilon \rightarrow 0}\left(\frac{i}{2 \pi \epsilon^{2}}\right)\left[\operatorname{tr}\left[\Gamma^{0} \notin\right]+i \mu \epsilon_{z} \operatorname{tr}\left[\Gamma^{5} \Gamma^{0} \notin\right]+O\left(\epsilon^{3}\right)\right] \\
& =2 \lim _{\epsilon \rightarrow 0}\left[\frac{i \epsilon_{0}}{\pi \epsilon^{2}}-\frac{\mu \epsilon_{z}^{2}}{\pi \epsilon^{2}}\right] \text {. }
\end{aligned}
$$

We take the symmetric limit to preserve Lorentz symmetry in the ultraviolet regime,

$$
\lim _{\epsilon \rightarrow 0} \frac{\epsilon_{\mu} \epsilon_{\nu}}{\epsilon^{2}}=\frac{g_{\mu \nu}}{2}
$$


The first term is odd in $\sim \epsilon_{0}$, and so vanishes after averaging over both directions. Hence

$$
\lim _{\epsilon \rightarrow 0}\left\langle\bar{\Phi}(x+\epsilon) \Gamma^{0} \Phi(x)\right\rangle_{\mu \neq 0}=\frac{\mu}{\pi} .
$$

This equals the baryon number density for a gas of two flavors of free quarks, with chemical potential $\mu$.

The computation can be repeated for other operators, such as $\langle\bar{\Phi} \Phi\rangle,\left\langle\bar{\Phi} \Gamma^{5} \Phi\right\rangle$, and $\left\langle\bar{\Phi} \Gamma^{z} \Phi\right\rangle$. These operators do not have anomalous terms, since

$$
\operatorname{tr}\left[\Gamma^{5} \Gamma^{5} \notin\right]=0, \quad \operatorname{tr}\left[\Gamma^{5} \notin\right]=0, \quad \frac{\epsilon_{z}}{\epsilon^{2}} \operatorname{tr}\left[\Gamma^{5} \Gamma^{z} \notin\right]=\frac{\epsilon_{z} \epsilon_{0}}{\epsilon^{2}} \rightarrow 0,
$$

respectively. For these condensates, the explicit phase dependence, in transforming from $\Phi$ to $\Phi^{\prime}$, must be taken into account, but there are no additional terms from the anomaly.

\section{References}

[1] G. 't Hooft, Nucl. Phys. B 72 (1974) 461.

[2] S. Coleman, $1 / N_{\mathrm{c}}$, Aspects of Symmetry, Sec. 8, Proceedings of the International School of Subnuclear Physics, 17th Erice, 1979; E. Witten, Nucl. Phys. B 160 (1979) 57; A. V. Manohar, arXiv:hep-ph/9802419].

[3] M. Teper, PoS LAT2008 (2008) 022; M. Panero, Phys. Rev. Lett. 103 (2009) 232001.

[4] G. 't Hooft, Nucl. Phys. B 75 (1974) 461.

[5] C.G. Callan, Jr., N. Coote, and D.J. Gross, Phys. Rev. D 13 (1976) 1649; I. Bars and M. B. Green, ibid. 17 (1978) 537; A. R. Zhitnitsky, Phys. Lett. B 165 (1985) 405; F. Lenz, M. Thies, K. Yazaki and S. Levit, Annals Phys. 208 (1991) 1; L. L. Salcedo, S. Levit and J. W. Negele, Nucl. Phys. B 361 (1991) 585; M. Engelhardt, Phys. Lett. B 355 (1995) 507; A. R. Zhitnitsky, Phys. Rev. D 53 (1996) 5821.

[6] L. McLerran and R. D. Pisarski, Nucl. Phys. A 796 (2007) 83.

[7] Y. Hidaka, L. D. McLerran and R. D. Pisarski, Nucl. Phys. A 808 (2008) 117; K. Fukushima, Phys. Rev. D 77 (2008) 114028; [Erratum-ibid. D 78 (2008) 039902]; L. McLerran, K. Redlich and C. Sasaki, ibid. 824 (2009) 86; A. Andronic et al., arXiv:0911.4806]; R. Stock, F. Becattini, T. Kollegger, M. Mitrovski and T. Schuster, arXiv:0911.5705.

[8] L. Y. Glozman and R. F. Wagenbrunn, Phys. Rev. D 77 (2008) 054027; L. Y. Glozman, ibid. 79 (2009) 037504; ibid. 80 (2009) 037701. 
[9] P. Guo and A. P. Szczepaniak, Phys. Rev. D 79 (2009) 116006.

[10] V.N. Gribov, Nucl. Phys. B 139 (1978) 1; Y. L. Dokshitzer and D. E. Kharzeev, Ann. Rev. Nucl. Part. Sci. 54 (2004) 487.

[11] D. Zwanziger, Phys. Rev. Lett. 90 (2003) 102001; Phys. Rev. D 69 (2004) 016002 .

[12] M. G. Alford, A. Schmitt, K. Rajagopal and T. Schafer, Rev. Mod. Phys. 80 (2008) 1455.

[13] M. T. Frandsen, C. Kouvaris and F. Sannino, Phys. Rev. D 74 (2006) 117503; M. I. Buchoff, A. Cherman and T. D. Cohen, arXiv:0910.0470; T. D. Cohen, D. L. Shafer and R. F. Lebed, Phys. Rev. D 81 (2010) 036006.

[14] A. Tsvelik, Quantum Field Theory in Condensed Matter Physics (Cambridge University Press, Cambridge, 2003).

[15] D. V. Deryagin, D. Y. Grigoriev and V. A. Rubakov, Intl. Jour. Mod. Phys. A 7 (1992) 659.

[16] E. Shuster and D. T. Son, Nucl. Phys. B 573 (2000) 434.

[17] B. Y. Park, M. Rho, A. Wirzba and I. Zahed, Phys. Rev. D 62 (2000) 034015; R. Rapp, E. V. Shuryak and I. Zahed, ibid. 63 (2001) 034008; B. Bringoltz, J. High Energy Phys. 016 (2007) 0703; S. Maedan, arXiv:0908.0594.

[18] W. Fischler, J. B. Kogut and L. Susskind, Phys. Rev. D 19 (1979) 1188; S. T. Love, ibid. 23 (1981) 420; Y. C. Kao and Y. W. Lee, ibid. 50 (1994) 1165; H. R. Christiansen and F. A. Schaposnik, ibid. 53 (1996) 3260; ibid. 55 (1997) 4920; S. Nagy, J. Polonyi and K. Sailer, ibid. 70 (2004) 105023; P. de Forcrand and U. Wenger, PoS LAT2006 (2006) 152; M. A. Metlitski, ibid. 75 (2007) 045004; S. G. Maciel and S. Perez, ibid. 78 (2008) 065005.

[19] V. Schon and M. Thies, Phys. Rev. D 62 (2000) 096002; M. Thies, ibid. 69 (2004) 067703; M. Thies and K. Urlichs, ibid. 67 (2003) 125015; ibid. 71 (2005) 105008; ibid. 72 (2005) 105008; O. Schnetz, M. Thies and K. Urlichs, Annals Phys. 321 (2006) 2604; C. Boehmer, M. Thies and K. Urlichs, Phys. Rev. D 75 (2007) 105017. G. Basar and G. V. Dunne, Phys. Rev. Lett. 100 (2008) 200404; Phys. Rev. D 78 (2008) 065022; C. Boehmer, U. Fritsch, S. Kraus and M. Thies, ibid. 78 (2008) 065043; G. Basar, G. V. Dunne and M. Thies, ibid. 79 (2009) 105012; C. Boehmer and M. Thies, arXiv:0912.2664.

[20] B. Bringoltz, Phys. Rev. D 79 (2009) 105021; ibid. 79 (2009) 125006.

[21] K. Y. Kim, S. J. Sin and I. Zahed, arXiv:hep-th/0608046]; N. Horigome and Y. Tanii, J. High Energy Phys. 072 (2007) 0701; A. Parnachev and D. A. Sahakyan, Nucl. Phys. B 768 (2007) 177; S. Nakamura, Y. Seo, S. J. Sin and K. P. Yogendran, J. Korean Phys. Soc. 52 (2008) 1734; S. Kobayashi, D. Mateos, S. Matsuura, R. C. Myers and R. M. Thomson, J. High Energy Phys. 016 (2007) 0702; D. Yamada, J. High Energy Phys. 020 (2008) 0810; O. Bergman, G. Lifschytz and M. Lippert, ibid. 0711 (2007) 056; M. Rozali, 
H. H. Shieh, M. Van Raamsdonk and J. Wu, ibid. 0801 (2008) 053; K. Ghoroku, M. Ishihara and A. Nakamura, Phys. Rev. D 76 (2007) 124006; A. Parnachev, J. High Energy Phys. 062 (2008) 0802; A. Karch and A. O'Bannon, J. High Energy Phys. 074 (2007) 0711; D. Mateos, S. Matsuura, R. C. Myers and R. M. Thomson, ibid. 0711 (2007) 085; M. Rozali, H. H. Shieh, M. Van Raamsdonk and J. Wu, ibid. 0801 (2008) 053; K. Y. Kim, S. J. Sin and I. Zahed, ibid. 0809 (2008) 001; O. Bergman, G. Lifschytz and M. Lippert, Phys. Rev. D 79 (2009) 105024; K. Hashimoto, Prog. Theor. Phys. 121 (2009) 241; K. Nawa, H. Suganuma and T. Kojo, Phys. Rev. D 79 (2009) 026005; K-Y. Kim and I. Zahed, J. High Energy Phys. 075 (2008) 0812; M. Kulaxizi and A. Parnachev, Nucl. Phys. B 815 (2009) 125; A. Rebhan, A. Schmitt and S. A. Stricker, J. High Energy Phys. 026 (2010) 1001; M. Rho, S. J. Sin and I. Zahed, [arXiv:0910.3774].

[22] A. B. Migdal, Zh. Eksp. Teor. Fiz. 61 (1971) 2209 [Sov. Phys. JETP 34 (1972) 1184]; Usp. Fiz. Nauk. 105 (1971) 781 [Sov. Phys. Usp. 14 (1972) 813]; Phys. Rev. Lett. 31 (1973) 257; Phys. Lett. B 47 (1973) 96; Rev. Mod. Phys. 50 (1978) 107; R. F. Sawyer, Phys. Rev. Lett. 29 (1972) 382; D. J. Scalapino, ibid. 29 (1972) 386; ibid. 31 (1973) 1556; R. F. Sawyer and D. J. Scalapino, Phys. Rev. D 7 (1973) 953; G. Baym, Phys. Rev. Lett. 30 (1973) 1340 A. B. Migdal, O. A. Markin and I. I. Mishustin, Sov. Phys. JETP 39 (1974) 212; R. F. Dashen and J. T. Manassah, Phys. Lett. B 50 (1974) 460; G. Baym, D. Campbell, R. F. Dashen and J. Manassah, Phys. Lett. B 58 (1975) 304; D. K. Campbell, R. F. Dashen and J. T. Manassah, Phys. Rev. D 12 (1975) 979; ibid. 12 (1975) 1010; A. B. Migdal, G. A. Sorokin, O. A. Markin and I. N. Mishustin, Phys. Lett. B 65 (1976) 423; A. B. Migdal, A. I. Chernoutsan and I. N. Mishustin, Phys. Lett. B 83 (1979) 158; W. Weise, Comments Nucl. Part. Phys. 10 (1981) 109; G. Baym, Nucl. Phys. A 352 (1981) 355; K. Kolehmainen and G. Baym, Nucl. Phys. A 382 (1982) 528; G. Baym, B. L. Friman and G. Grinstein, Nucl. Phys. B 210 (1982) 193; A. B. Migdal, E. E. Saperstein, M. A. Troitsky and D. N. Voskresensky, Phys. Rep. 192 (1990) 179; A. Akmal and V. R. Pandharipande, Phys. Rev. C 56 (1997) 2261; D. T. Son and M. A. Stephanov, Phys. Atom. Nucl. 64 (2001) 834 [Yad. Fiz. 64 (2001) 899]; A. Barducci, R. Casalbuoni, G. Pettini and L. Ravagli, Phys. Rev. D 71 (2005) 016011; E. Nakano and T. Tatsumi, Phys. Rev. D 71 (2005) 114006; D. Ebert and K. G. Klimenko, Jour. Phys. G 32 (2006) 599; J. O. Andersen, ibid. 75 (2007) 065011; H. Abuki, R. Anglani, R. Gatto, M. Pellicoro and M. Ruggieri, ibid. 79 (2009) 034032; T. Herpay and P. Kovacs, ibid. 78 (2008) 116008; D. Ebert and K. G. Klimenko, arXiv:0902.1861.

[23] D. B. Kaplan and A. E. Nelson, Phys. Lett. B 175 (1986) 57; H. D. Politzer and M. B. Wise, Phys. Lett. B 273 (1991) 156; G. E. Brown, V. Thorsson, K. Kubodera and M. Rho, Phys. Lett. B 291 (1992) 355; G. E. Brown, C. H. Lee, M. Rho and V. Thorsson, Nucl. Phys. A 567 (1994) 937; C. H. Lee, G. E. Brown, D. P. Min and M. Rho, Nucl. Phys. A 585 (1995) 401; P. J. Ellis, R. Knorren and M. Prakash, Phys. Lett. B 349 (1995) 11; N. K. Glendenning and J. Schaffner-Bielich, Phys. Rev. Lett. 81 (1998) 4564; Phys. Rev. C 60 (1999) 025803 ; M. M. Forbes, Phys. Rev. D 72 (2005) 094032.

[24] D. Nickel, Phys. Rev. Lett. 103 (2009) 072301; Phys. Rev. D 80 (2009) 074025. 
[25] J. Wess and B. Zumino, Phys. Lett. B 37 (1971) 95; S. Novikov, Usp. Mat. Nauk, 37 (1982) 3; E. Witten, Comm. Math. Phys. 92 (1984) 455.

[26] P. J. Steinhardt, Nucl. Phys. B 176 (1980) 100. A. R. Zhitnitsky, Phys. Lett. B 165 (1985) 405; I. Affleck, Nucl. Phys. B 265 (1986) 448; A. Ferrando and V. Vento, Phys. Lett. B 256 (1991) 503; L. L. Salcedo, S. Levit and J. W. Negele, Nucl. Phys. B 361 (1991) 585; E. Abdalla and M. C. B. Abdalla, Phys. Rep. 265 (1996) 253.

[27] P. Di Vecchia, B. Durhuus and J. L. Petersen, Phys. Lett. B 144 (1984) 245; D. Gonzales and A. N. Redlich, Phys. Lett. B 147 (1984) 150; Nucl. Phys. B 256 (1985) 621; J. L. Petersen, Acta Phys. Polon. B 16, (1985) 271; E. Abdalla and M.C.B. Abdalla, Nucl. Phys. B 255 (1985) 392. Y. Frishman and J. Sonnenschein, Nucl. Phys. B 294 (1987) 801; Phys. Rep. 223 (1993) 309; Y. Frishman, A. Hanany and M. Karliner, Nucl. Phys. B 424 (1994) 3; A. Armoni, Y. Frishman and J. Sonnenschein, ibid. 596 (2001) 459.

[28] R. D. Pisarski and D. H. Rischke, Phys. Rev. D 60 (1999) 094013.

[29] T. Kojo, R. D. Pisarski, and A. Tsvelik, manuscript in preparation.

[30] R. D. Pisarski, Phys. Rev. D 62 (2000) 111501(R); A. Dumitru and R. D. Pisarski, Phys. Lett. B 504 (2001) 282; ibid. 525 (2002) 95; A. Dumitru, Y. Hatta, J. Lenaghan, K. Orginos and R. D. Pisarski, Phys. Rev. D 70 (2004) 034511; A. Dumitru, J. Lenaghan and R. D. Pisarski, ibid. 71 (2005) 074004; R. D. Pisarski, ibid. 74 (2006) 121703(R); Y. Hidaka and R. D. Pisarski ibid. 78 (2008) 071501(R); ibid. 80 (2009) 036004; ibid. 80 (2009) 074504; arXiv:0912.0940.

[31] C. P. Korthals Altes, R. D. Pisarski and A. Sinkovics, Phys. Rev. D 61 (2000) 056007 . 